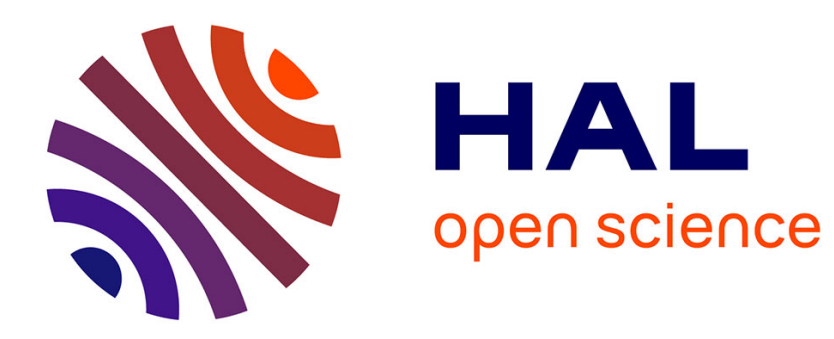

\title{
Compteurs Čerenkov à substances fluorescentes
}

\author{
G. Cosme, Sophie Jullian, J. Lefrançois
}

\section{To cite this version:}

G. Cosme, Sophie Jullian, J. Lefrançois. Compteurs Čerenkov à substances fluorescentes. Revue de Physique Appliquée, 1969, 4 (2), pp.297-298. 10.1051/rphysap:0196900402029700 . jpa-00243269

\section{HAL Id: jpa-00243269 https://hal.science/jpa-00243269}

Submitted on 1 Jan 1969

HAL is a multi-disciplinary open access archive for the deposit and dissemination of scientific research documents, whether they are published or not. The documents may come from teaching and research institutions in France or abroad, or from public or private research centers.
L'archive ouverte pluridisciplinaire HAL, est destinée au dépôt et à la diffusion de documents scientifiques de niveau recherche, publiés ou non, émanant des établissements d'enseignement et de recherche français ou étrangers, des laboratoires publics ou privés. 


\title{
GOMPTEURS ČERENKOV A SUBSTANGES FLUORESGENTES
}

\author{
G. COSME, S. JULLIAN et J. LEFRANÇOIS, \\ École Normale Supérieure, Laboratoire de l'Accélérateur Linéaire, Orsay.
}

\begin{abstract}
Résumé. - Nous avons étudié la possibilité d'utiliser des substances fluorescentes dissoutes dans différents radiateurs Čerenkov liquides. Le Popop dissous dans l'éthylène glycol ou le bromobenzène fournit un gain de lumière d'environ 2,5. Un compteur basé sur ces propriétés a été utilisé pour détecter les produits de désintégration du méson $\mathrm{K}^{+}$.
\end{abstract}

Abstract. - The use has been studied of light shifting substances dissolved in a liquid Cerenkov counter. It was found that Popop was the most suitable shifter studied. Ethylene glycol and bromobenzene were found to be good solvents. The light gain when using a shifter was approximately 2.5. A counter based on these properties was used to detect disintegration products of $\mathrm{K}^{+}$mesons.

Ce type de compteur Čerenkov a été mis au point dans le cadre d'une expérience de photoproduction de mésons $\mathrm{K}^{+}$. Dans les radiateurs Čerenkov sont dissoutes des substances fluorescentes qui absorbent la lumière Čerenkov ultraviolette et émettent ensuite isotropiquement. On peut alors détecter par effet Čerenkov le passage de particules quelles que soient les directions de celles-ci dans le radiateur (comme dans un scintillateur).

Les solutions utilisées doivent avoir les propriétés suivantes : la substance fluorescente doit absorber les radiations U.V. qui sont favorisées dans l'effet Čerenkov $\mathrm{I}(\lambda) \mathrm{d} \lambda \alpha\left(1-\frac{1}{\beta^{2} n^{2}(\lambda)}\right) \frac{\mathrm{d} \lambda}{\lambda^{2}}$ et émettre dans une bande de longueurs d'onde centrée sur la photocathode utilisée. Il faut donc que le radiateur utilisé soit transparent pour les faibles longueurs d'onde. L'indice $n(\lambda)$ des solvants doit aussi être compatible avec le $\beta$ des particules à détecter. La constante de temps $\tau$ d'émission de la fluorescence doit être assez courte (quelques ns) pour que le signal à l'anode du photomultiplicateur puisse être utilisé dans une électronique rapide. En effet, suivant la valeur de $\tau$, la lumière Čerenkov directe et la lumière de fluorescence peuvent arriver, sur la photocathode pendant un temps $\Delta \tau$. On aura donc à l'anode soit un signal intégré par le $\mathrm{PM}($ si $\tau \approx 1$ à $5 \mathrm{~ns})$, soit un signal lent non intégré (si $\tau \geqslant 15 \mathrm{~ns}$ ). On doit alors, dans ce dernier cas, intégrer le signal dans un circuit externe (avec une constante de temps longue) et le « clipper » ensuite pour avoir un signal rapide dont la hauteur est effectivement proportionnelle à la quantité de lumière vue par la photocathode.

Nous avons retenu les radiateurs suivants : l'eau plus le 2-amino-6,8... (60 mg/l) absorbe la lumière Čerenkov entre 2100 et $3800 \AA$ et émet entre 4200 et $5500 \AA$; le bromobenzène plus Popop (100 mg/l) absorbe entre
3000 et $4000 \AA$ et émet entre 4000 et $5500 \AA$. L'éthylène glycol plus Popop (solution saturée $\approx 4 \mathrm{mg} / \mathrm{l}$ ) absorbe entre 2100 et $4000 \AA$ et émet entre 4000 et $5500 \AA$ (fig. 1). Trois types de compteurs Čerenkov,

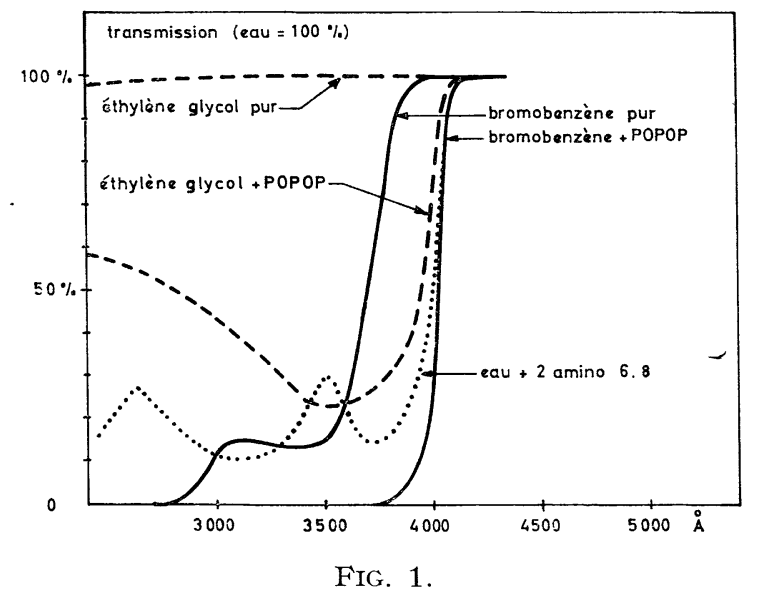

géométriquement identiques, ont été étudiés avec les solvants ci-dessus avec ou sans substances fluorescentes. Nous avons mesuré les gains de lumière obtenus avec l'addition de substances fluorescentes; les parois internes du compteur étaient aluminisées. Les résultats expérimentaux montrés dans le tableau I sont comparés à un calcul théorique donnant la quantité de lumière $Q$ reçue par la photocathode dans les différents cas :

$$
Q=\int_{\lambda_{1}}^{\lambda_{2}} I(\lambda) \mathrm{d} \lambda
$$

$\lambda_{1}$ et $\lambda_{2}$ étant les longueurs d'onde limites permises à la fois par la transparence du milieu et par la photocathode utilisée. On suppose en particulier que l'effi- 
TABLEAU I

\begin{tabular}{|c|c|c|c|c|c|}
\hline & $\underline{\beta}$ & $\begin{array}{c}\text { SANS } \\
\text { INTÉGRATION } \\
-\end{array}$ & $\begin{array}{c}\text { AVEG } \\
\text { INTÉGRATION } \\
-\end{array}$ & Théorie & $\begin{array}{c}\text { THÉORIE EXP. } \\
\text { AVEG } \\
\text { INTÉGRATION } \\
-\end{array}$ \\
\hline Bromobenzène & 0,94 & 1,63 & 1,68 & 1,81 & 1,08 \\
\hline$+100 \mathrm{mg} / 1$ Popop & 0,76 & 2,35 & 2,40 & 1,85 & 0,77 \\
\hline Éthylène glycol & 0,94 & 2,05 & 2,20 & 2,47 & 1,12 \\
\hline+4 mg/l Popop & 0,76 & 2,72 & 3,16 & 2,60 & 0,82 \\
\hline $\begin{array}{c}\text { Eau } \\
+2 \text {-amino-6,8... }\end{array}$ & 0,94 & 1,49 & $\begin{array}{ll}\text { (1) } & 2,30 \\
\text { (2) } & 2,63 \\
\text { (3) } & 2,74\end{array}$ & 3,14 & 1,14 \\
\hline
\end{tabular}

cacité de la fluorescence est 1 , que le pouvoir réflecteur des parois aluminisées est 1 et que la sensibilité de la photocathode est constante dans la bande de longueur d'onde choisie.

La différence entre valeurs théoriques et expérimentales est due aux approximations faites dans ce calcul. Le 2-amino-6,8... a une constante de fluorescence longue $(\simeq 15 \mathrm{~ns})$; pour détecter la lumière de fluorescence, on doit intégrer le signal.

Nous avons construit pour notre expérience de photoproduction de mésons $\mathrm{K}^{+}$deux compteurs Čerenkov : l'un, à bromobenzène plus Popop comme compteur d'anticoïncidence à seuil, détectait efficacement des mésons $\pi^{+}$dont le $\beta$ variait de 0,7 à 0,9 . L'utilisation du Popop facilitait la détection de l'effet Čerenkov perpendiculairement à la direction de la particule. L'autre, à éthylène glycol plus Popop, détectait les mésons $\pi^{+}, \mu^{+}$et les électrons de désintégration du $\mathrm{K}^{+}$ à l'arrêt émis isotropiquement $\left(\beta_{\mu^{+}}=0,9, \beta_{\pi^{+}}=0,8\right.$, $\left.\beta_{\mathrm{e}^{+}}=1\right)$. On ne détectait donc que $88 \%$ des mésons pendant un temps correspondant à deux vies moyennes du $\mathrm{K}^{+}$. L'efficacité d'un tel compteur était de $87 \%$ (pour un seuil du discriminateur électronique compatible avec le bruit de fond propre à notre expérience).
Pour augmenter la quantité de lumière sur la photocathode du PM (60 AVP), on recueillait à la fois la lumière en réflexion totale et la lumière en réflexion métallique grâce à une double paroi ( fig. 2).

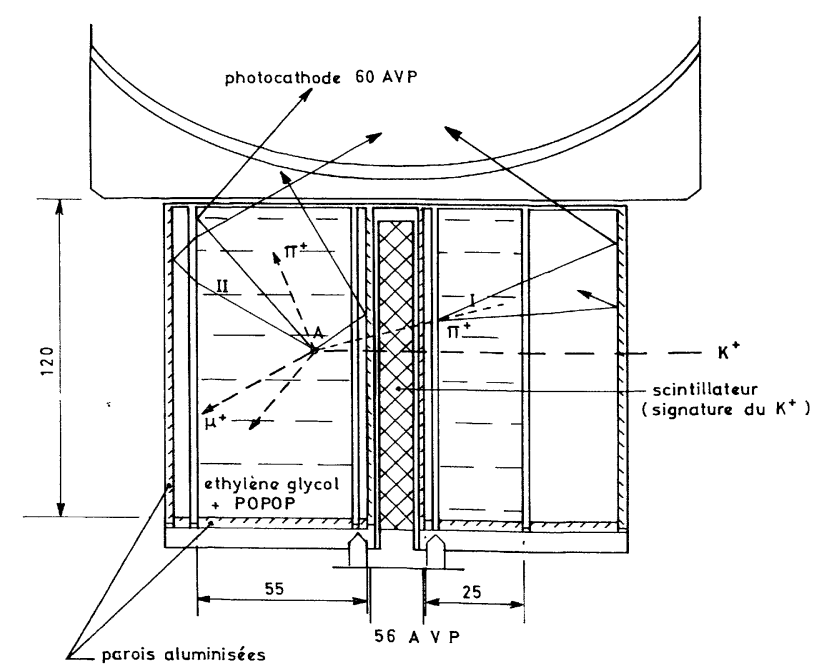

FIG. 2. - A, zone d'arrêt du méson $\mathrm{K}^{+}$. 